\title{
QUASI-ORDERINGS AND TOPOLOGIES ON FINITE SETS
}

\author{
HENRY SHARP, JR.
}

1. Throughout this paper $S$ is the finite set $\left\{s_{1}, s_{2}, \cdots, s_{n}\right\}$, and if $J$ is a topology on $S$ then $A^{-}$denotes the $J$-closure of the subset $A$ of $S$. It is our purpose to investigate topologies on $S$ and to answer a few combinatorial questions related to these topologies. The connection between $T_{0}$-topologies and partial orderings on finite sets (Theorem 7) already appears in several standard references [1, p. 28] and $[2$, p. 14]. That there is a one-to-one correspondence between the topologies on $S$ and the quasi-orderings on $S$ follows from the next paragraph.

For each set $A \subset S, A^{-}=\bigcup\left\{s_{i}\right\}$ - over all $s_{i} \in A$, hence to identify a topology on $S$ it suffices to display the closures of all singletons. For this purpose we choose the relation matrix

$$
\begin{aligned}
t_{i j} & =1, & & \text { if } s_{j} \in\left\{s_{i}\right\}^{-}, \\
& =0, & & \text { otherwise. }
\end{aligned}
$$

The Kuratowski closure axioms $[3$, p. 43$]$ imply that $\left[t_{i j}\right]$ is reflexive $\left(A \subset A^{-}\right)$and transitive $\left(A^{--}=A^{-}\right)$.

Let $T=\left[t_{i j}\right]$ be the matrix corresponding to a topology $J$ and let $F_{i}$ and $B_{j}$ be the subsets of $S$ having characteristic functions $\left\{\left(s_{1}, t_{i 1}\right),\left(s_{2}, t_{i 2}\right), \cdots,\left(s_{n}, t_{i n}\right)\right\}$ and $\left\{\left(s_{1}, t_{1 j}\right),\left(s_{2}, t_{2 j}\right), \cdots,\left(s_{n}, t_{n j}\right)\right\}$. Note that $s_{j} \in F_{i}$ iff $s_{i} \in B_{j}$. For each $i, F_{i}=\left\{s_{i}\right\}$ - is the minimal closed set containing $s_{i}$.

THEOREM 1. For each $j, B_{j}$ is the minimal open set in 3 containing $s_{j}$.

Proof. We show first that $S-B_{j}$ is closed. If $s_{i} \in S-B_{j}$ and if $s_{k} \in F_{i}$, then $t_{i j}=0$ and $t_{i k}=1$. Transitivity forbids $t_{k j}=1$, hence $F_{i} \subset S-B_{j}$. To show that $B_{j}$ is minimal, let $U$ be any open set containing $s_{j}$. If $s_{k} \in S-U$ then $F_{k} \subset S-U$ and $s_{j} \notin F_{k}$. Hence $s_{k} \notin B_{j}$ and $S-U \subset S-B_{j}$.

COROLlary. The weight $[1$, p. 7] of any topology on $S$ does not exceed $n+1$.

Adjoining $\varnothing$ to the family of distinct minimal open sets $B_{j}$ produces a basis for the topology which we call the minimal basis.

THEOREM 2. If $i \neq j, t_{i j}=1$ iff $B_{i} \subset B_{j}$.

Received by the editors July 29, 1964. 
Proof. If $B_{i} \subset B_{j}$ then $s_{i} \in B_{j}$ and $t_{i j}=1$. On the other hand suppose $t_{i j}=1$. For each $k$ if $t_{k i}=1$ then $t_{k j}=1$ and $B_{i} \subset B_{j}$.

Corollary. If $i \neq j, t_{i j}=t_{j i}=1$ iff $B_{i}=B_{j}$.

Theorem 3. If $i \neq j, t_{i j}=1$ iff $F_{j} \subset F_{i}$.

The proof is like that of Theorem 2.

Corollary. If $i \neq j, t_{i j}=t_{j i}=1$ iff $F_{j}=F_{i}$.

Theorem 4. A reflexive, $n \times n$, zero-one matrix $T$ corresponds to a topology on $S$ iff $T^{2}=T$.

Proof. Matrix multiplication here involves Boolean arithmetic. The theorem follows from the fact that a reflexive relation $\rho$ is transitive iff $\rho \rho=\rho[2$, p. 209].

2. Let $J$ and $J^{*}$ be topologies on $S$ with corresponding matrices $T=\left[t_{i j}\right]$ and $T^{*}=\left[t_{i j}^{*}\right]$. Then $J=J^{*}$ iff $t_{i j}=t_{i j}^{*}$ for each $i$ and $j$. On the other hand $J^{*}$ and $J^{*}$ are topologically equivalent iff there exists a permutation $\pi(S)=S$ under which the minimal bases of $J$ and $\Im^{*}$ correspond. The matrices $T$ and $T^{*}$ are called isomorphic (nonisomorphic) if $J^{3}$ and $J^{*}$ are equivalent (nonequivalent) [5]. It follows that $T$ and $T^{*}$ are isomorphic iff there exists an $n \times n$ permutation matrix $P$ such that $T^{*}=P^{\prime} T P$, where $P^{\prime}$ is the transpose of $P$.

If $J$ is a topology on $S$ then the family $J^{\prime}$ of complements of members of $J$ also is a topology on $S$. We shall call $J^{\prime}$ the transpose (or the $d u a l)$ topology with respect to $\mathfrak{J}$.

Theorem 5. If $T$ is the matrix corresponding to the topology $J$ then $T^{\prime}$ (the transpose of $T$ ) is the matrix corresponding to the topology $J^{\prime}$.

Proof. We show first that $\left(T^{\prime}\right)^{2}=T^{\prime}$. Let $T=\left[t_{i j}\right]$ and $T^{\prime}=\left[t_{j i}\right]$. Then $\left(T^{\prime}\right)^{2}=\left[v_{i j}\right]$ where

$$
\tau_{i j}=\sum_{k=1}^{n} t_{j k} t_{k i}
$$

But $T^{2}=T$, therefore $v_{i j}=t_{j i}$ and $\left(T^{\prime}\right)^{2}=T^{\prime}$. By Theorem 4, $T^{\prime}$ corresponds to a topology on $S$, and the nonempty members of its minimal basis are the 3 -closures $F_{i}$. Hence the topology consists of the family of all unions $U F_{i}$; that is, of all J-closed sets.

Theorem 6. The topology $\mathrm{J}$ is not connected iff for some $k, 0<k<n$, both $T$ and $T^{\prime}$ contain the same $k \times(n-k)$ zero submatrix.

Proof. A topology $\mathfrak{J}$ is not connected iff there exists a nonempty proper subset $A$ of $S$ such that $A \in J$ and $A \in J^{\prime}$. This means that 
$A=\mathrm{U} B_{i}=\bigcup F_{i}$ over all $i$ such that $s_{i} \in A$. But the complement, $S-A$, has the same property. Let $k$ be the cardinal of $A$ and the theorem follows.

In finite topological spaces the separation properties characterizing $T_{0^{-}}, T_{1^{-}}, T_{2^{-}}$, etc., spaces are of limited help in the study of topological structure. The only interesting partition of topologies in this hierarchy occurs at the $T_{0}$ level. The theorem stated next formalizes the relation mentioned at the beginning of the paper.

THEOREM 7. The topology J on $S$ is $T_{0}$ iff its matrix $T$ is anti-symmetric (that is, $T$ defines a partial ordering on $S$ ).

Corollary. The weight of a topology $J$ on $S$ is $n+1$ iff $J$ is $T_{0}$.

In general, the topologies $J$ and $J^{\prime}$ are neither equal nor equivalent. In the event, however, that $J^{\prime}=J$ the matrix $T$ is symmetric and we call its corresponding topology symmetric. The symmetric topologies correspond to the equivalence relations on $S$. Theorems 6 and 7 imply that $J^{\prime}$ is $T_{0}$ or connected iff $J$ is.

In the matrix $T$ corresponding to the topology $\mathfrak{I}$, let $C(\mathcal{F})$ $=\left(c_{1}, c_{2}, \cdots, c_{n}\right)$ be the column sum vector and let $R(\Im)=\left(r_{1}, r_{2}\right.$, $\left.\cdots, r_{n}\right)$ be the row sum vector $[4$, p. 61]. The class of vectors each of which is some permutation of the coordinates of $C$ (or of $R$ ) is a topological invariant. Also, the sum, $\tau$, of the entries in $T$ is a topological invariant. These, unfortunately, are not topological characters; for the two matrices below describe nonequivalent topologies.

$$
\left[\begin{array}{llllll}
1 & 0 & 0 & 0 & 0 & 0 \\
0 & 1 & 0 & 0 & 0 & 0 \\
0 & 1 & 1 & 0 & 0 & 0 \\
1 & 0 & 0 & 1 & 0 & 0 \\
1 & 0 & 0 & 0 & 1 & 0 \\
1 & 1 & 1 & 0 & 0 & 1
\end{array}\right] \quad\left[\begin{array}{llllll}
1 & 0 & 0 & 0 & 0 & 0 \\
0 & 1 & 0 & 0 & 0 & 0 \\
1 & 0 & 1 & 0 & 0 & 0 \\
0 & 1 & 0 & 1 & 0 & 0 \\
1 & 0 & 0 & 0 & 1 & 0 \\
1 & 1 & 1 & 0 & 0 & 1
\end{array}\right] .
$$

In each matrix $C=(4,3,2,1,1,1)$ and $R=(1,1,2,2,2,4)$.

We shall call the matrix $T=\left[t_{i j}\right]$ triangular if $t_{i j}=0$ for all $i<j$.

THEOREM 8. The matrix T corresponding to a topology 3 is isomorphic to a triangular matrix iff $\mathrm{J}$ is $T_{0}$.

Proof. If $T$ is isomorphic to a triangular matrix then $t_{i j} \cdot t_{j i}=0$ for all $i \neq j$. Now assume that $J$ is $T_{0}$. There exists a permutation matrix $P$ such that $T^{*}=P^{\prime} T P$ has a monotone (nonincreasing) column sum vector. If $T^{*}$ is not triangular, then for some $i<j t_{i j}^{*}=1$. By Theorem 2 
$B_{i}^{*} \subset B_{j}^{*}$, and by the Corollary to Theorem $7 B_{i}^{*} \neq B_{j}^{*}$, hence $c_{i}<c_{j}$ which is a contradiction.

Theorem 9. Let $\mathfrak{J}$ be a topology on $S$. There exists a topology $\mathfrak{J}^{*}$ equivalent to $\mathfrak{J}$ such that $C\left(\mathfrak{J}^{*}\right)$ and $R\left(\mathrm{~J}^{*}\right)$ each are monotone (nonincreasing) iff $\mathfrak{J}$ is symmetric.

Proof. Sufficiency is evident since $c_{i}=r_{i}$. If $J$ is not symmetric then for some $i \neq j t_{i j}=1$ while $t_{j i}=0$. By Theorems 2 and $3 c_{i} \leqq c_{j}$ and $r_{i} \geqq r_{j}$, but since $t_{j i}=0$ strict inequality holds in each case.

Theorem 10. Among the symmetric topologies only the discrete is $T_{0}$ and only the indiscrete is connected.

Proof. If $t_{i j}=t_{j i}=1$ and if $J$ is $T_{0}$ then by Theorem $7 i=j$. To prove the latter statement, we may assume by Theorem 9 that the column sum and row sum vectors are monotone. The least coordinate in the column sum vector is $c_{n}$, and we assume that $c_{n}=k<n$. If $t_{i n}=1$ then $B_{i}=B_{n}$ and $T$ contains $k$ identical columns each with $n-k$ zero entries. By Theorem $6 T$ is not connected.

The following corollary refers to different, although possibly homeomorphic, topologies.

Corollary. If $n>1$ then the number of different $T_{0}$ topologies is odd, the number of different connected topologies is odd, and the number of connected $T_{0}$ topologies is even [6].

3. If $n$ is 3 the trivial topologies (discrete and indiscrete) correspond, respectively, to the matrices

$$
\left[\begin{array}{lll}
1 & 0 & 0 \\
0 & 1 & 0 \\
0 & 0 & 1
\end{array}\right] \text { and }\left[\begin{array}{lll}
1 & 1 & 1 \\
1 & 1 & 1 \\
1 & 1 & 1
\end{array}\right] \text {. }
$$

It is evident that the extreme values of $\tau$, in general, are $n$ and $n^{2}$; but it is not the case that all intermediate values are possible.

TheOREM 11. If $J$ is nontrivial then $n<\tau \leqq n^{2}-n+1$.

Proof. Only the right-hand part of the inequality is in question. Suppose for some $i \neq j t_{i j}=0$. Then for each $k$ such that $k \neq i$ and $k \neq j$ either $t_{i k}=0$ or $t_{k j}=0$.

A little more than 10 years ago R. L. Davis published a formula (among others) for the number of nonisomorphic reflexive relations on $S$ [5]. The author is not aware of a formula enumerating the subfamily of transitive relations. Such a formula, in addition to being of value in logic and combinatorics, would answer the question: how many nonequivalent topologies are there on a finite set? 
For small $n$ the preceding theory can be used to good advantage in the enumeration problem. Though the method lacks subtlety, it is not impossibly tedious for $n \leqq 5$, even without the assistance of a digital computer. In Table 1 , " $t$ " denotes the number of nonequivalent topologies on $S$, " $t c$ " denotes the number that are connected, " $t o$ " denotes the number that are $T_{0}$, "tco" denotes the number that are both connected and $T_{0}$, and " $t s$ " denotes the number that are symmetric. Figure 1 displays matrices corresponding to all nonequivalent topologies for $n=3$ and $n=4$.

TABLE 1

\begin{tabular}{l|rrrrr}
\hline$n$ & $t$ & $t c$ & $t o$ & $t c o$ & $t s$ \\
\hline 2 & 3 & 2 & 2 & 1 & 2 \\
3 & 9 & 6 & 5 & 3 & 3 \\
4 & 33 & 21 & 16 & 10 & 5 \\
5 & 139 & 94 & 63 & 44 & 7 \\
\hline
\end{tabular}

Figure 1

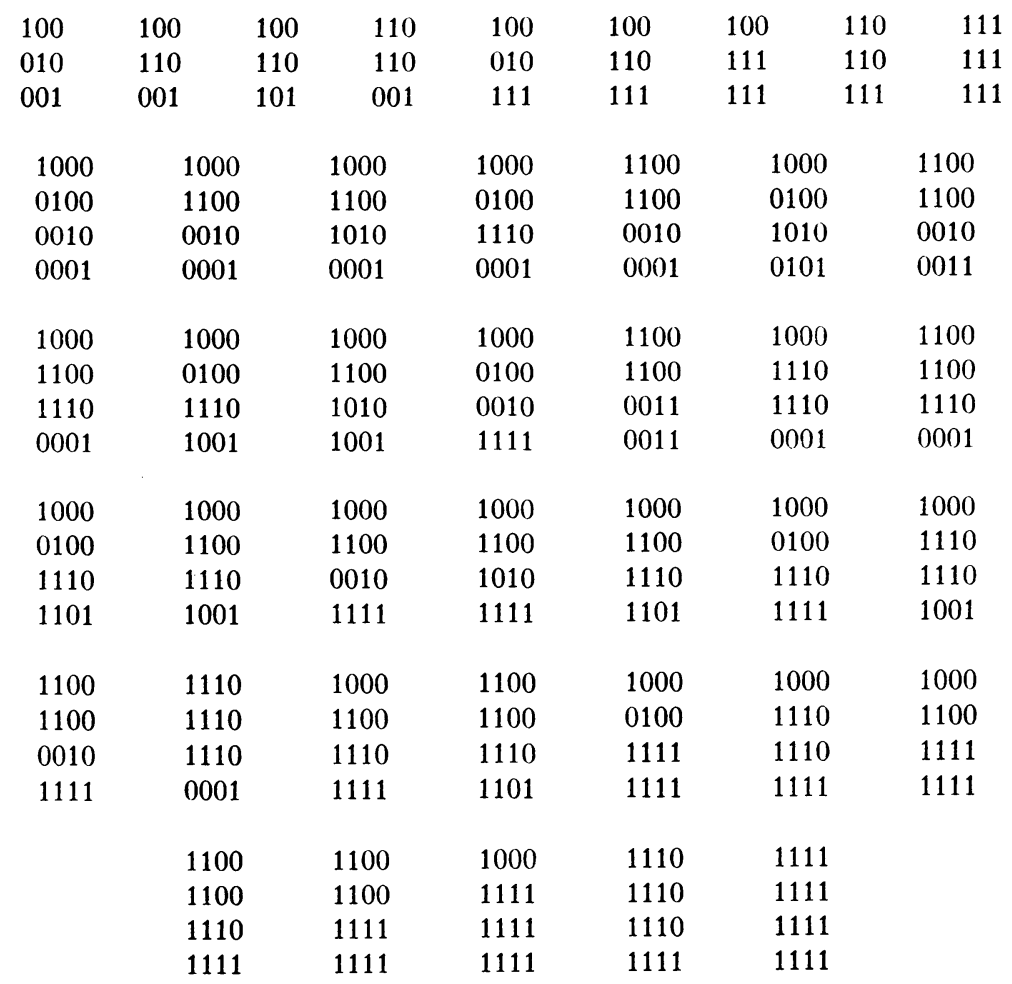




\section{REFERENCES}

1. P. S. Aleksandrov, Combinatorial topology, Vol. 1, Graylock, Rochester, N. Y., 1956.

2. Garrett Birkhoff, Lattice theory (rev. ed.), Amer. Math. Soc. Colloq. Publ. Vol. 25, Amer. Math. Soc., Providence, R. I., 1948.

3. John L. Kelley, General topology, Van Nostrand, New York, 1955.

4. Herbert John Ryser, Combinatorial mathematics, The Carus Mathematical Monographs, No. XIV, Math. Assoc. Amer., 1963.

5. Robert L. Davis, The number of structures of finite relations, Proc. Amer. Math. Soc. 4 (1953), 486.

6. R. A. Rankin, Problem No. 5137, Amer. Math. Monthly 70 (1963), 898.

EMORY UNIVERSITY 